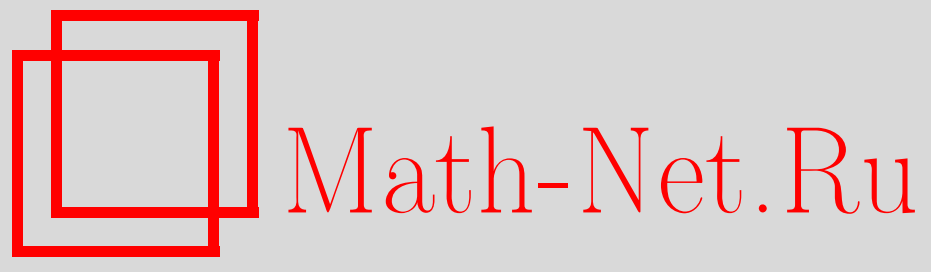

Р. В. Иванов, О дискретной аппроксимации опционов Американского типа, УМН, 2006, том 61, выпуск 1, 179 180

DOI: https://doi.org/10.4213/rm1693

Использование Общероссийского математического портала Math-Net.Ru подразумевает, что вы прочитали и согласны с пользовательским соглашением http: //www . mathnet.ru/rus/agreement

Параметры загрузки:

IP: 3.89 .185 .249

26 апреля 2023 г., 02:34:46

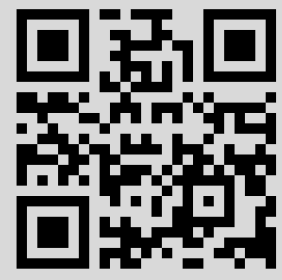




\section{О дискретной аппроксимации опционов Американского типа}

\section{Р. В. Иванов}

В непрерывной модели Блэка-Шоулса (см. [1])

$$
\begin{aligned}
d B_{t} & =r B_{t} d t, & & r \geqslant 0, \\
d S_{t} & =S_{t}\left(r d t+\sigma d W_{t}\right), & & \sigma>0, \quad t \in[0, T],
\end{aligned}
$$

решение задачи хеджирования опционов Американского типа (см. [2; разделы VIII.2, VIII.3]) в случае конечного временного горизонта $T$ неизвестно. Автором предлагается аппроксимация цен опционов данного типа с помощью дискретной биномиальной $\triangle$-модели

$$
\begin{gathered}
g B_{n \triangle}-B_{(n-1) \triangle}=r \triangle B_{(n-1) \triangle}, \\
S_{n \triangle}-S_{(n-1) \triangle}=\left(e^{\sigma \varepsilon_{n}^{\Delta}}-1\right) S_{(n-1) \triangle},
\end{gathered}
$$

где $\varepsilon_{n}^{\triangle}, n=1, \ldots,[T / \triangle],-$ независимые одинаково распределенные случайные величины, принимающие значения $\pm \sqrt{\triangle}$, а $\triangle>0$ есть шаг модели. В дискретном случае цена может быть найдена с помощью метода обратной индукции (см. [2; разделы VI.2, VI.5, VIII.3]). Рассматриваются стандартные опционы с функциями выплат $f_{t}=e^{-\lambda t} g\left(S_{t}\right)$, где $g\left(S_{t}\right)=\left(S_{t}-K\right)^{+}$(опцион покупателя) или $g\left(S_{t}\right)=\left(K-S_{t}\right)^{+}$ (опцион продавца) и $\lambda \geqslant 0$. В дискретном случае полагаем $f_{n \triangle}=(1+\lambda \triangle)^{-n} g\left(S_{n \triangle}\right)$. Пусть $S_{0}=x, V(x, T)$ - цена опциона в непрерывной модели, а $V(x, T, \triangle)$ - в дискретной с шагом $\triangle$.

Теорема 1. Пусть в модели Блэка-Шоулса $r<\sigma^{2} / 2$. Тогда существуют такие положительные константы $C$ и с, зависящие только от параметров модели, что при $T \geqslant C \log \triangle^{-1}$ для всех достаточно малых $\triangle>0$

$$
|V(x, T)-V(x, T, \triangle)| \leqslant c \triangle .
$$

Доказательство. Обозначим $V(x)=V(x, \infty)$ и $V(x, \triangle)=V(x, \infty, \triangle)$. Пусть $\tau^{*}$ есть оптимальный момент остановки в задаче хеджирования в модели Блэка-Шоулса на бесконечном временном интервале, т.е.

$$
V(x)=\mathrm{E} e^{-(r+\lambda) \tau^{*}} g\left(S_{\tau^{*}}\right) I\left\{\tau^{*}<\infty\right\}
$$

(см. [2; раздел VIII.2]), а $\mathfrak{M}_{0}^{T}$ - множество всех моментов остановки, принимающих значения на интервале $[0, T]$. Тогда

$$
\begin{aligned}
V(x, T) & =\sup _{\tau \in \mathfrak{M}_{0}^{T}} \mathrm{E} e^{-(\lambda+r) \tau} g\left(S_{\tau}\right) \\
& \geqslant \mathrm{E} e^{-(\lambda+r) \min \left(\tau^{*}, T\right)} g\left(S_{\min \left(\tau^{*}, T\right)}\right) \geqslant \mathrm{E} e^{-(\lambda+r) \tau^{*}} g\left(S_{\tau^{*}}\right) I\left\{\tau^{*} \leqslant T\right\},
\end{aligned}
$$

и, следовательно,

$$
V(x)-V(x, T) \leqslant \mathrm{E} e^{-(\lambda+r) \tau^{*}} g\left(S_{\tau^{*}}\right) I\left\{T<\tau^{*}<\infty\right\} .
$$

Аналогично, для $\triangle$-дискретной модели

$$
V(x, \triangle)-V(x, T, \triangle) \leqslant \mathrm{E}[(1+\lambda \triangle)(1+r \triangle)]^{-\tilde{\tau}} g\left(S_{\tilde{\tau}}\right) I\{T<\tilde{\tau}<\infty\},
$$

где $\tilde{\tau}$ есть оптимальный момент остановки в $\triangle$-дискретной модели с бесконечным временным горизонтом (см. [2; раздел VI.5]). Отсюда следует, что для некоторой константы $c_{0}>0$

$$
|V(x, T)-V(x, T, \triangle)| \leqslant|V(x)-V(x, \triangle)|+c_{0} \max \left\{\mathrm{P}\left\{T<\tau^{*}<\infty\right\}, \mathrm{P}\{T<\tilde{\tau}<\infty\}\right\} .
$$


Устремляя $\triangle$ к нулю, нетрудно получить, что

$$
|V(x)-V(x, \triangle)| \leqslant c \triangle
$$

для всех достаточно малых $\triangle>0$ при некотором $c>0$. Используя метод [3; теорема 3] для оценки второго слагаемого в правой части неравенства (1), окончательно имеем

$$
|V(x, T)-V(x, T, \triangle)| \leqslant c \triangle+c_{1} e^{-c_{2} T}
$$

для всех достаточно малых $\triangle>0$ с некоторыми положительными константами $c, c_{1}$, $c_{2}$, зависящими только от параметров модели. Из (2) следует утверждение теоремы.

Теорема 2. Пусть в модели Блэка-Шоулса $r=\sigma^{2} / 2$. Тогда для некоторых положительных констант $C$ и с, зависящих только от параметров модели, при $T \geqslant C \triangle^{-1}$ для всех достаточно малых $\triangle>0$

$$
|V(x, T)-V(x, T, \triangle)| \leqslant c \triangle^{\frac{1}{2}} .
$$

ДоказАтельство. Аналогично (1), имеем

$$
|V(x, T)-V(x, T, \triangle)| \leqslant c \triangle+c_{1} \max \left\{P\left\{T<\tau^{*}<\infty\right\}, \mathrm{P}\{T<\tilde{\tau}<\infty\}\right\} .
$$

В данном случае оптимальные моменты $\tau^{*}$ и $\tilde{\tau}$ являются конечными (см. $[2$; разделы VI.5, VIII.2]), и нам необходимо оценить

$$
\max \left\{\mathrm{P}\left\{\tau^{*}>T\right\}, \mathrm{P}\{\tilde{\tau}>T\}\right\} .
$$

Используя неравенство Берри-Эссеена, окончательно получаем аналогично (2)

$$
|V(x, T)-V(x, T, \triangle)| \leqslant c \triangle+\frac{c_{1}}{\sqrt{T}}+\frac{c_{2}}{\sqrt{[T / \triangle]}}
$$

для всех достаточно малых $\triangle>0$.

Теорема 3. Пусть в модели Блэка-Шоулса $r>\sigma^{2} / 2$. Тогда для некоторых положительных констант $C$ и с, зависящих только от параметров модели, при $T \geqslant C \triangle^{-1}$ для всех достаточно малых $\triangle>0$

$$
|V(x, T)-V(x, T, \triangle)| \leqslant c \triangle .
$$

ДокАзАТЕльство. Следуя методу доказательства предыдущей теоремы, устанавливаем, что

$$
|V(x, T)-V(x, T, \triangle)| \leqslant c \triangle+\frac{c_{1}}{\sqrt{T}} e^{-c_{2} T}+\frac{c_{3}}{\sqrt{[T / \triangle]}}
$$

с некоторыми положительными константами $c, c_{1}, c_{2}, c_{3}$, зависящими только от параметров модели. Отсюда следует утверждение теоремы.

Автор благодарен профессору А.Н. Ширяеву, который инициировал написание этой заметки.

\section{Список литературы}

[1] F. Black, M. Scholes, J. Polit. Econ., 81:3 (1973), 637-654. [2] А. Н. Ширяев, Основы стохастической финансовой математики, Фазис, М., 1998. [3] А. А. Новиков, А. Н. Ширяев, Теория вероятн. и ее примен., 49:2 (2004), 373-382.

P. В. Иванов (R. V. Ivanov)

Московский государственный университет им. М. В. Ломоносова

E-mail: roma_ivanov@rambler.ru
Представлено А. В. Булинским Принято редколлегией 19.12.2005 